\title{
Análise da eficiência na produção de energia eólica nos principais estados brasileiros produtores, por meio da aplicação de análise por envoltória de dados
}

\author{
Analysis of the efficiency of wind energy production in the main \\ brazilian producting states by data envelopment analysis
}

\author{
Marcus Vinícius Sousa \\ Rodrigues \\ Universidade Federal Rural \\ do Semi-Árido (UFERSA) \\ - Campus Angicos (Angicos \\ $-\mathrm{RN}$ ) \\ E-mail:marcus@ufersa. \\ edu.br
}

Marisete Dantas de Aquino Universidade Federal do Ceará (UFC) - Campus do

Pici (Fortaleza-CE)

E-mail: marisete@ufc.br

\section{Antônio Clécio Fontelles Thomaz \\ Universidade Estadual do Ceará (UECE) - Campus do Itaperi (Fortaleza - CE) \\ E-mail: clecio@larces.uece. br}

\begin{abstract}
Resumo
A energia eólica é uma energia produzida pela força dos ventos, sendo considerada limpa e renovável. Múltiplos são os fatores que podem ser utilizados para analisar a eficiência na produção de eletricidade a partir dos ventos. O objetivo principal deste artigo de pesquisa consiste em avaliar a eficiência relativa da geração eólica nos principais estados brasileiros produtores, durante o ano de 2014, por meio de análise por envoltória de dados. Das onze unidades avaliadas, quatro foram consideradas eficientes e sete, não eficientes. Em seguida, o modelo da fronteira invertida foi usado de modo a ordenar as unidades. Além disso, o método da fronteira invertida identificou duas unidades eficientes apresentando uma falsa eficiência. Assim, a produção de energia eólica do Estado do Piauí se apresentou como a unidade mais eficiente de todo o conjunto analisado, em contraste com o Estado de Pernambuco, que foi considerado como o menos eficiente de todos.
\end{abstract}

Palavras-chave: Energia eólica. Análise envoltória de dados. Eficiência relativa.

\begin{abstract}
Wind energy is energy produced by the force of the winds, and it is considered clean and renewable. There are many factors that can be used to analyze the efficiency in the production of electricity from wind, in the main Brazilian states. The main objective of this research paper is to assess the relative efficiency of wind generation in the main producing Brazilian states in 2014, by applying data envelopment analysis (DEA). Four out of eleven units assessed were considered efficient and seven non efficient. Then the inverted frontier model was used to order the units according to the measurement of an efficiency index, as defined by normalized efficiency. Furthermore, the inverted frontier method identified two efficient units as having a false efficiency. Thus, wind energy production of the state of Piauí was presented as the most efficient unit of the whole analyzed, in contrast to the state of Pernambuco's, which was considered the least efficient of all.
\end{abstract}

Keywords: Wind power; Data envelopment analysis; Relative efficiency.

\section{Introdução}

A energia eólica é uma energia produzida pela força dos ventos, sendo considerada como uma das fontes de energia mais antigas usadas pela humanidade. É uma energia considerada limpa e renovável, sendo proveniente da radiação solar, uma vez que os ventos são gerados pelo aquecimento não uniforme da superfície terrestre. A conversão da energia eólica em energia mecânica consiste em uma técnica relativamente simples, bastando apenas que se tenha um potencial eólico disponível e que se resista aos caprichos da natureza. As primeiras turbinas eólicas aplicadas para converter a energia dos ventos em energia elétrica surgem no final do século XIX, com o rápido crescimento do uso da eletricidade no planeta. 
Segundo Carneiro et al. (2013), dentre os vários benefícios do uso da energia eólica para geração de energia elétrica, pode-se citar que ela não apresenta riscos de radiação, apresenta fonte livre, além de produzir alguns dos menores riscos de poluição para a atmosfera ou para os recursos hídricos.

A aplicação das energias consideradas renováveis para geração de energia elétrica, tais como a energia eólica, só teve um forte desenvolvimento a partir da crise petrolífera que ocorreu nos anos 1970 (Hamdan et al., 2014).

Em 1992, é instalada a primeira turbina eólica no Brasil, em Fernando de Noronha, apresentando as seguintes características: um gerador com uma potência de $75 \mathrm{~kW}$, rotor de 17 metros de diâmetro e torre de 23 metros de altura. Entretanto, para Fadigas (2011), a preocupação com o uso da energia eólica para geração de eletricidade pode ser considerada bem recente no Brasil, ou seja, o país só voltou suas atenções para esse recurso renovável e abundante apenas a partir de 2002 .

O desenvolvimento da energia eólica, no Brasil, tem ocorrido de forma gradual e consistente, porém a matriz elétrica brasileira é predominantemente hidrelétrica, aproveitando à vocação natural no Brasil. A ampliação da participação da geração eólica nessa matriz possibilitará uma maior autonomia energética em função do efeito complementar que a fonte eólica possui. (Kniess et al., 2013).

Atualmente, pode-se afirmar que o uso da energia eólica como fonte energética já é uma realidade no Brasil, entretanto, para que seja explorada em larga escala, torna-se necessário políticas de incentivo de modo a criar condições para uma maior participação dessa fonte na matriz energética brasileira. $\mathrm{O}$ equipamento que converte a energia cinética contida nos ventos (ou energia eólica) em energia elétrica é chamado de turbina eólica, ou aerogerador.

O aumento do interesse pelo uso da energia é́lica no mundo se deve, principalmente, ao crescente aumento da eficiência dos aerogeradores. Os países que mais utilizam essa fonte para geração de eletricidade são Alemanha, Espanha e Estados Unidos (Neumeister \& Silva, 2014).

A avaliação do desempenho entre unidades de atividades, como escolas, hospitais, bancos, concessionárias elétricas etc., é uma grande preocupação para o setor de administração, e pode-se dizer que, nos últimos anos, houve um forte crescimento no desenvolvimento de ferramentas para realizar essa tarefa (Athanassopoulos, 2012).

Múltiplos são os critérios que podem ser utilizados para analisar o desempenho (ou eficiência) da conversão da energia eólica em energia elétrica nos estados brasileiros. Contudo, uma análise como essa requer o uso de uma metodologia científica chamada de análise multicritério. A técnica de análise multicritério adotada nesta pesquisa é a análise por envoltória de dados (AED), também conhecida como DEA (sigla em inglês de Data Envelopment Analysis). Ela pode ser empregada em um conjunto de unidades de produção, comumente chamadas de unidades tomadoras de decisão (UTD), também chamada de DMU (sigla em inglês de Decision Making Unit), de modo a classificá-las de acordo com as suas performances.

Para Banker (1993), a AED pode ser definida como uma técnica de programação matemática que permite avaliar o grau de desempenho (ou eficiência) de um conjunto de unidades semelhantes, sendo considerados os recursos de que se dispõe com os produtos alcançados.

Além da eficiência, essa metodologia é capaz de determinar metas a serem almejadas para unidades ineficientes. Uma UTD pode representar qualquer conjunto de organizações ou departamentos que realizam fundamentalmente a mesma tarefa, com o mesmo conjunto de fatores (Adler \& Yazhemsky, 2010).

O objetivo principal deste artigo de pesquisa consiste em avaliar a eficiência relativa da produção de energia eólica nos principais estados brasileiros produtores, durante o ano de 2014, por meio da aplicação de análise por envoltória de dados. Em seguida, pretende-se ordenar as unidades avaliadas através da medida do índice de eficiência, obtido por meio do modelo da fronteira invertida.

\section{Referencial Teórico}

\subsection{Geração de energia eólica}

Uma turbina eólica pode ser definida como sendo um equipamento que converte parte da energia cinética contida nos ventos em energia elétrica. De acordo com o eixo ao redor do qual as pás da turbina giram, as turbinas eólicas são classificadas em: turbina eólica de eixo horizontal (TEEH) e turbina eólica de eixo vertical (TEEV). 
Essa conversão segue duas etapas: 1) primeiramente na turbina, que remove a porção da energia cinética disponível para conversão em energia mecânica, e 2) no gerador, que recebe a energia mecânica e a converte em energia elétrica, que é transmitida para a rede concessionária. (Pinto, 2014).

Atualmente, a maioria das turbinas eólicas em uso nos parques eólicos, para geração de energia elétrica, apresenta eixo de rotação na posição horizontal. Essas turbinas são compreendidas quase que exclusivamente sob as bases do conceito das hélices. Esse design, que inclui os moinhos de vento europeus e as máquinas eólicas modernas, é o princípio dominante na tecnologia eólica da atualidade.

As pás da turbina de eixo horizontal são fixadas por meio de flanges, em uma estrutura metálica denominada de cubo. Juntos, as pás e o cubo formam o rotor eólico do aerogerador. As pás podem ser entendidas como "motores a vento", pois são elas que interagem diretamente com o vento. A interação entre as pás de uma turbina eólica com os ventos que passam através da área do rotor deve ser a maior possível, de modo que a extração de potência seja tão eficiente quanto possível.

Por meio da Figura 1, é possível observar uma carcaça montada sobre a torre da turbina eólica, logo atrás do rotor eólico, onde se localizam o gerador elétrico, a caixa de engrenagens e todo o sistema de controle, chamado de nacele.

Figura 1 - Rotor eólico de uma turbina eólica de eixo vertical.

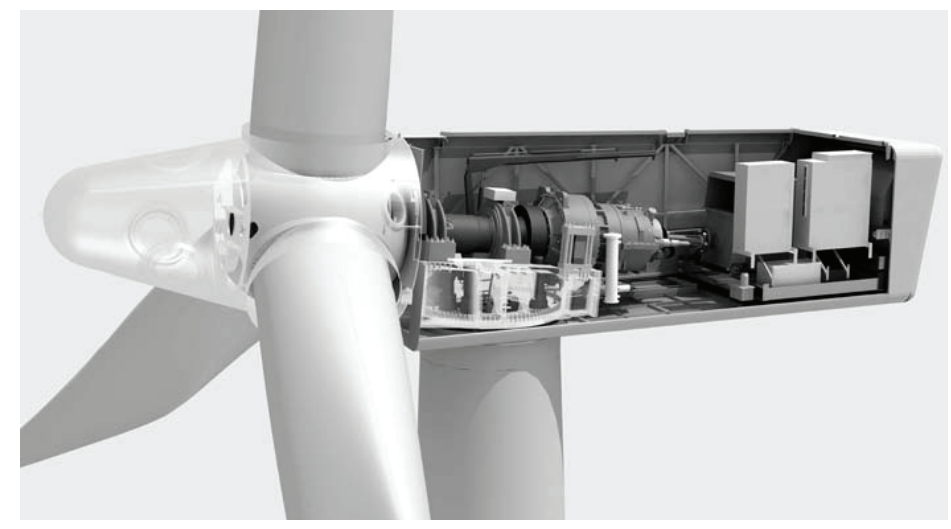

Fonte: Hansen, 2008

Segundo Pires \& Oliveira (2010), as pás podem ser consideradas como a parte mais importante do rotor eólico, e o tamanho delas (e, consequentemente, o diâmetro do rotor) está diretamente relacionado ao desempenho de um aerogerador.

Conforme Shamshirband et al. (2014), a energia cinética do vento captada pelo aerogerador é função da velocidade do vento, da massa específica do ar, da área de varredura das pás e ainda da altura em que o rotor está instalado.

A potência total disponível pelo vento, , em W (J/s), é dada pela seguinte expressão:

$$
\dot{\mathrm{W}}_{\text {disp }}=\frac{1}{2} \rho \mathrm{AV}^{3}
$$

Em que $\rho$ é a massa específica do ar em $\mathrm{kg} / \mathrm{m}^{3}$, A é a área de varredura das pás em rotação em $\mathrm{m}^{2} \mathrm{e} V$ é a velocidade do vento em $\mathrm{m} / \mathrm{s}$.

É importante salientar que apenas um percentual dessa energia, dada pela equação (1), é absorvida pelo rotor eólico do aerogerador. A potência extraída, , em W (J/s), pela turbina eólica é dada por

$$
\dot{\mathrm{W}}_{\mathrm{ext}}=\frac{1}{2} \mathrm{C}_{\mathrm{P}} \rho \mathrm{AV}^{3}
$$

Em que $\mathrm{C}_{\mathrm{P}}$ é o coeficiente de potência adimensional e representa o desempenho aerodinâmico dessa máquina (relação entre a potência mecânica do conversor eólico e a potência contida no vento).

Albert Betz, em 1920, demonstrou que a potência máxima que pode ser extraída do vento representa 59,3\% da potência total disponível no vento. Assim, o coeficiente de potência máximo, indicado por $\mathrm{C}_{\mathrm{P} \text {, máx }}$ e igual a 0,593 , é 
conhecido como número de Betz e serve como parâmetro ideal (ou um limite superior), com o qual se comporta o desempenho efetivo de uma turbina eólica. Entretanto, segundo Sharma \& Patel (2015), na prática, as turbinas eólicas modernas apresentam um rendimento em torno de $40 \%$.

Como dito anteriormente, uma turbina eólica capta uma parte da energia cinética do vento que atravessa a área varrida pelas pás do rotor e a transforma em energia mecânica de rotação. Em seguida, o eixo do rotor acionando o gerador elétrico transforma uma parte desta energia mecânica de rotação em energia elétrica.

A potência elétrica gerada, , em W (J/s), é também função do cubo da velocidade do vento, sendo dada por:

$$
\dot{\mathrm{W}}_{\text {elet }}=\frac{1}{2} \eta \mathrm{C}_{\mathrm{P}} \rho \pi \mathrm{R}^{2} \mathrm{~V}^{3}
$$

Em que $\eta$ é a eficiência da turbina eólica e R representa o raio do rotor eólico em $\mathrm{m}$. Vale salientar que a eficiência da turbina eólica pode ser entendida como o rendimento considerando as perdas no conjunto das transmissões mecânicas e as perdas no gerador elétrico.

\subsection{Análise por envoltória de dados}

Em um processo produtivo, os insumos empregados na produção devem ser utilizados da melhor forma possível, de modo que não hajam excessos. Assim, a medida de produtividade pode ser avaliada para comparar o desempenho (ou eficiência) entre várias unidades produtivas.

O conceito de eficiência está relacionado à comparação de produtividade entre várias unidades, sendo, portanto uma medida relativa. A medida de eficiência de uma UTD pode ser obtida por meio de uma análise detalhada da relação existente entre insumos e produtos. Esta relação é denominada retornos de escala. (Rodrigues et al., 2015).

A técnica de AED permite a realização de uma análise da eficiência comparativa de um conjunto de UTDs, demonstrando como cada unidade está operando em relação às demais do grupo, obtendo, desse modo, um diagnóstico completo de todo o conjunto (Barbosa \& Bastos, 2014).

Uma das grandes vantagens da AED é o fato de que não há a exigência de uma relação funcional entre insumos e produtos, como as abordagens tradicionais de estatística, tais como a regressão linear. Segundo Parker et al. (2015), a AED é usada para construir uma fronteira linear por partes, chamada de fronteira eficiente, usando para isso os fatores de insumo e de produto de cada unidade avaliada, e em seguida apresenta uma medida de eficiência relativa para cada unidade.

Para Rodrigues et al. (2015), "as melhores relações produtos/insumos são consideradas mais eficientes, estando situadas na fronteira eficiente, enquanto as menos eficientes estarão situadas na região abaixo dessa curva, denominada de envoltória convexa".

As unidades avaliadas em AED são comparadas conforme o conceito de eficiência de Farrel, que é definido como a razão entre a soma ponderada dos produtos e a soma ponderada dos insumos de cada unidade. Matematicamente, a eficiência para $\mathrm{m}$ insumos e s produtos, é dada pela seguinte expressão

$$
\text { Eficiência }=\sum_{i=1}^{s} U_{i} Y_{i} / \sum_{i=1}^{m} V_{j} X_{j}
$$

Em que $\mathrm{U}_{\mathrm{i}}$ é o peso do i-ésimo produto, $\mathrm{Y}_{\mathrm{i}}(\mathrm{i}=1, \ldots, \mathrm{s})$; e $\mathrm{V}_{\mathrm{j}}$ é o peso do $\mathrm{j}$-ésimo insumo, $\mathrm{X}_{\mathrm{j}}(\mathrm{j}=1, \ldots, \mathrm{m})$.

Assim, as unidades tidas como eficientes (chamadas de benchmarks) servem de referência para outras unidades consideradas ineficientes. Dessa forma, as unidades ineficientes estabelecem metas a serem alcançadas com a finalidade de melhorarem as suas performances. As unidades que se apresentam abaixo da fronteira de eficiência, tidas como ineficientes, conforme ilustrada na Figura 2, devem ser projetadas em direção à fronteira eficiente, alcançado assim as suas parceiras eficientes. 
Figura 2 - Fronteira de eficiência, UTDs eficientes e ineficientes.

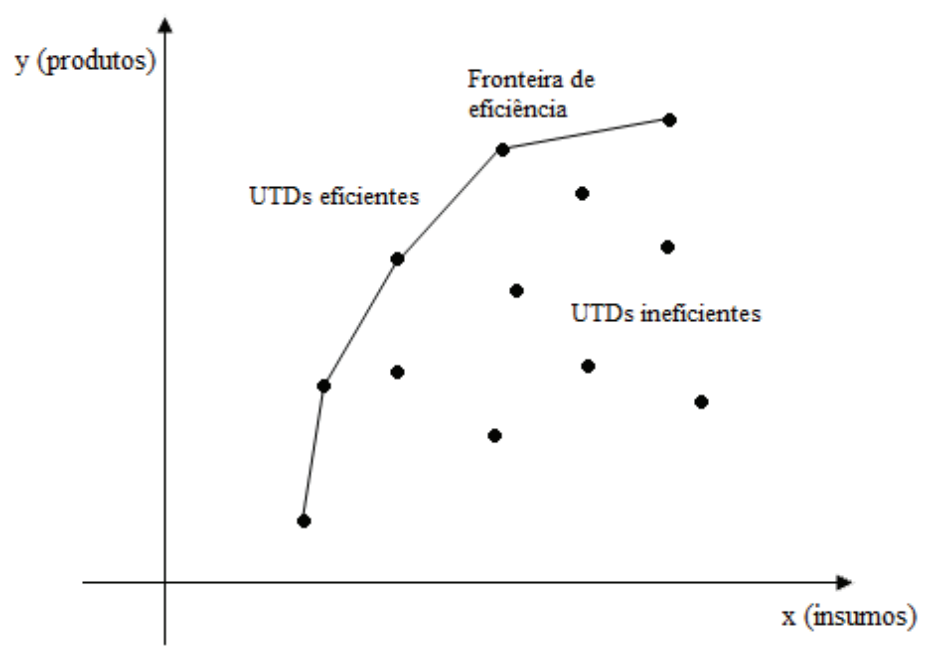

Fonte: Rodrigues, 2014.

Uma UTD ineficiente pode atingir a fronteira eficiente de duas formas distintas: por meio da minimização das entradas (insumos), mantendo constantes as saídas (produtos), chamada de orientação a insumo; e por meio da maximização das saídas (produtos), mantendo constantes as entradas (insumos), chamada de orientação a produto.

Segundo Périco et al. (2008):

O estudo sobre eficiência de qualquer organização passa, necessariamente, pela questão da fronteira eficiente. Essa fronteira representa um máximo de produtividade para uma determinada quantia de recursos estabelecida, significando que, quanto menor a quantia, melhor a produtividade. Em suma, estarão mais próximas da fronteira de eficiência aquelas organizações que alcançarem maior produtividade, consumindo menos recursos.

Existem dois modelos básicos de AED: o modelo CCR, também conhecido como CRS (sigla em inglês Constant Return to Scale), que admite retornos de escala constante; e o modelo BCC, também conhecido como VRS (sigla em inglês de Variable Return to Scale), que admite retornos variáveis de escala.

\subsubsection{Modelo CCR}

A metodologia AED teve início com o trabalho publicado por Charnes et al. (1978), que avaliou a eficiência de programas escolares no Estado do Texas, Estados Unidos, ficando conhecido como modelo CCR (iniciais dos autores: Charnes, Cooper e Rhodes) e sendo desenvolvido originalmente com uma orientação a insumo.

O modelo CCR trabalha com retornos constantes de escala e o cálculo da eficiência de um modelo AED pode usar tanto medidas orientadas a insumo como medidas orientadas a produto.

Logo, suponha que existam $n$ UTDs, semelhantes entre si, utilizando $m$ insumos e $s$ produtos. Assim, em um modelo CCR, com orientação a insumo, os pesos dos insumos e dos produtos de uma unidade objeto, $\mathrm{UTD}_{\mathrm{O}}$, são determinados através da resolução do seguinte problema de programação matemática,

$$
\max E_{f O}=\sum_{i=1}^{s} U_{i} Y_{i o}
$$

Sujeito a:

$$
\begin{aligned}
& \sum_{j=1}^{m} V_{j} X_{j O}=1 \\
& \sum_{i=1}^{s} U_{i} Y_{i k}-\sum_{j=1}^{m} V_{j} X_{j k} \leq 0, \quad(k=1, \ldots, n) \\
& U_{i} \geq 0(i=1, \ldots, s) ; \quad V_{j} \geq 0(j=1, \ldots, m)
\end{aligned}
$$


Em que $\mathrm{E}_{\mathrm{fo}}$ é a eficiência relativa da $\mathrm{UTD}_{\mathrm{O}} ; \mathrm{Y}_{\mathrm{ik}}$ e $\mathrm{X}_{\mathrm{jk}}$ são as quantidades de produto observado i da unidade $\mathrm{k}$ e de insumo observado $\mathrm{j}$ da unidade $\mathrm{k}$, respectivamente; $\mathrm{U}_{\mathrm{i}} \mathrm{e} \mathrm{V}_{\mathrm{j}}$ são os pesos dados ao produto i e ao insumo $\mathrm{j}$, respectivamente; e, $\mathrm{Y}_{\mathrm{io}}$ e $\mathrm{X}_{\mathrm{io}}$ são as quantidades do produto i e do insumo $\mathrm{j}$ da unidade objeto, respectivamente.

O problema apresentado nas equações (5) e (6) é comumente conhecida como Modelo dos Multiplicadores, também chamado de Primal, com orientação a insumo. O nome "orientação a insumo" vem do fato de que a eficiência é alcançada por intermédio da redução dos insumos (também chamados de recursos).

Em seguida, serão apresentadas as formulações do modelo CCR orientado a produto. Vale lembrar que nesse tipo de orientação as saídas (produtos) são maximizadas, enquanto as entradas (insumos) permanecem inalteradas. Assim, a modelagem dos multiplicadores para o modelo CCR, orientado a produto, é dada da seguinte forma:

$$
\min \mathrm{h}_{\mathrm{o}}=\sum_{\mathrm{i}=1}^{\mathrm{m}} \mathrm{V}_{\mathrm{j}} \mathrm{X}_{\mathrm{jo}}
$$

Sujeito a:

$$
\begin{aligned}
& \sum_{\mathrm{i}=1}^{\mathrm{s}} \mathrm{U}_{\mathrm{i}} \mathrm{Y}_{\mathrm{iO}}=1 \\
& \sum_{\mathrm{i}=1}^{\mathrm{s}} \mathrm{U}_{\mathrm{i}} \mathrm{Y}_{\mathrm{ik}}-\sum_{\mathrm{j}=1}^{\mathrm{m}} \mathrm{V}_{\mathrm{j}} \mathrm{X}_{\mathrm{jk}} \leq 0, \quad(\mathrm{k}=1, \ldots, \mathrm{n}) \\
& \mathrm{U}_{\mathrm{i}} \geq 0(\mathrm{i}=1, \ldots, \mathrm{s}) ; \mathrm{V}_{\mathrm{j}} \geq 0(\mathrm{j}=1, \ldots, \mathrm{m})
\end{aligned}
$$

Conforme Gomes et al. (2012), o modelo multiplicador trata da relação das somas ponderadas de produtos e insumos com os pesos escolhidos de modo a se tornar mais favorável a cada UTD analisada.

É importante enfatizar que na modelagem AED dos multiplicadores, tanto com orientação a insumo como com orientação a produto, as incógnitas de decisão são os pesos $U_{i}$ e $V_{j}$. Assim, pode-se afirmar que o conjunto dos pesos encontrados para cada UTD analisada deve ser tal que a medida da eficiência seja máxima.

\subsubsection{Modelo BCC}

O modelo foi desenvolvido por Banker et al. (1984), em que os retornos de escala são considerados variáveis, sendo conhecido como modelo BCC em homenagem aos autores (Banker, Charnes e Cooper).

O modelo passa a admitir tecnologias com retornos variáveis de escala, o que resulta em uma fronteira formada por combinações convexas de unidades eficientes. Segundo Gomes Júnior et al. (2013), esse modelo adota o axioma da convexidade em vez do axioma da proporcionalidade do modelo CCR.

A suposição de retornos constantes de escala do modelo CCR é relaxada para retornos de escala variáveis no modelo BCC, por intermédio da adição de uma variável livre, para orientação a insumo, e para orientação a produto.

Assim, o modelo BCC, orientado a insumo pode ser dado pelo seguinte problema:

$$
\max \mathrm{E}_{\mathrm{fO}}=\sum_{\mathrm{i}=1}^{\mathrm{s}} \mathrm{U}_{\mathrm{i}} \mathrm{Y}_{\mathrm{iO}}+\mathrm{U}_{*}
$$

Sujeito a:

$$
\begin{aligned}
& \sum_{\mathrm{j}=1}^{\mathrm{m}} \mathrm{V}_{\mathrm{j}} \mathrm{X}_{\mathrm{jO}}=1 \\
& \sum_{\mathrm{i}=1}^{\mathrm{s}} \mathrm{U}_{\mathrm{i}} \mathrm{Y}_{\mathrm{ik}}-\sum_{\mathrm{j}=1}^{\mathrm{m}} \mathrm{V}_{\mathrm{j}} \mathrm{X}_{\mathrm{jk}}+\mathrm{U}_{\mathrm{s}} \leq 0, \quad(\mathrm{k}=1, \ldots, \mathrm{n}) \\
& \mathrm{U}_{\mathrm{i}} \geq 0(\mathrm{i}=1, \ldots, \mathrm{s}) ; \mathrm{V}_{\mathrm{j}} \geq 0(\mathrm{j}=1, \ldots, \mathrm{m}) ; \mathrm{U}_{\mathrm{s}} \in \mathbb{R}
\end{aligned}
$$

Se a variável livre for positiva, o modelo apresenta rendimentos de escala não decrescente (RND); se a variável for negativa, o modelo se apresenta com rendimentos de escala não crescente (RNC).

A modelagem AED dos multiplicadores para o modelo BCC, orientado a produto, é dada da seguinte forma: 


$$
\min \mathrm{h}_{\mathrm{o}}=\sum_{\mathrm{j}=1}^{\mathrm{m}} \mathrm{V}_{\mathrm{j}} \mathrm{X}_{\mathrm{jo}}+\mathrm{V}_{\mathrm{s}}
$$

Sujeito a:

$$
\begin{aligned}
& \sum_{\mathrm{i}=1}^{\mathrm{s}} \mathrm{U}_{\mathrm{i}} \mathrm{Y}_{\mathrm{io}}=1 \\
& \sum_{\mathrm{i}=1}^{\mathrm{s}} \mathrm{U}_{\mathrm{i}} \mathrm{Y}_{\mathrm{ik}}-\sum_{\mathrm{j}=1}^{\mathrm{m}} \mathrm{V}_{\mathrm{j}} \mathrm{X}_{\mathrm{jk}}+\mathrm{V}_{\mathrm{x}} \leq 0, \quad(\mathrm{k}=1, \ldots, \mathrm{n}) \\
& \mathrm{U}_{\mathrm{i}} \geq 0(\mathrm{i}=1, \ldots, \mathrm{s}) ; \mathrm{V}_{\mathrm{i}} \geq 0(\mathrm{j}=1, \ldots, \mathrm{m}) ; \mathrm{V}_{\mathrm{s}} \in \mathbb{R}
\end{aligned}
$$

Se a variável livre for positiva, o modelo apresenta rendimentos de escala não crescente (RNC); se for negativa, o modelo se apresenta com rendimentos de escala não decrescente (RND).

\section{METODOLOGIA}

\subsection{Unidades tomadoras de decisão (UTDs)}

Segundo Rodrigues (2014), uma metodologia AED para analisar a eficiência de um conjunto de UTDs se apresenta como as seguintes etapas:

seleção das UTDs;

definição dos fatores de avaliação (insumos e produtos);

escolha e aplicação da modelagem de AED;

análise e interpretação dos resultados obtidos.

O Brasil tem uma característica interessante com relação à produção eólica. Como a velocidade dos ventos costuma ser maior em períodos de estiagem, é possível então que os parques eólicos operem complementando a geração hidroelétrica, o que consequentemente manteria parte da água nos reservatórios nos períodos de seca ou de pouca chuva. $\mathrm{O}$ aumento da geração eólica instalada pode reduzir a dependência do Nordeste de outras fontes de energia (térmicas ou transferidas de outras regiões) no período de seca. (Pinto, 2014).

A região Nordeste se destaca das demais regiões do país em relação ao potencial eólico, e os estados do Rio Grande do Norte e do Ceará apresentam os maiores potenciais eólicos. É estimado que em toda a extensão dos litorais do Rio Grande do Norte e do Ceará apresentam em conjunto um potencial eólico de aproximadamente 12,0 GW, correspondendo a cerca de $25,0 \%$ da oferta energética do Brasil.

O Brasil tem uma das matrizes energéticas mais limpas do mundo. Conforme ilustrado na Figura 3, as fontes renováveis representam mais de $70 \%$ da matriz nacional, sendo $66,6 \%$ hidráulica, 3,7\% eólica e menos de $0,1 \%$ solar. A capacidade instalada total de geração de energia elétrica do Brasil atingiu 134.008 MW em janeiro de 2015 (Brasil, 2015).

Figura 3 - Matriz energética brasileira em potência instalada em janeiro de 2015.

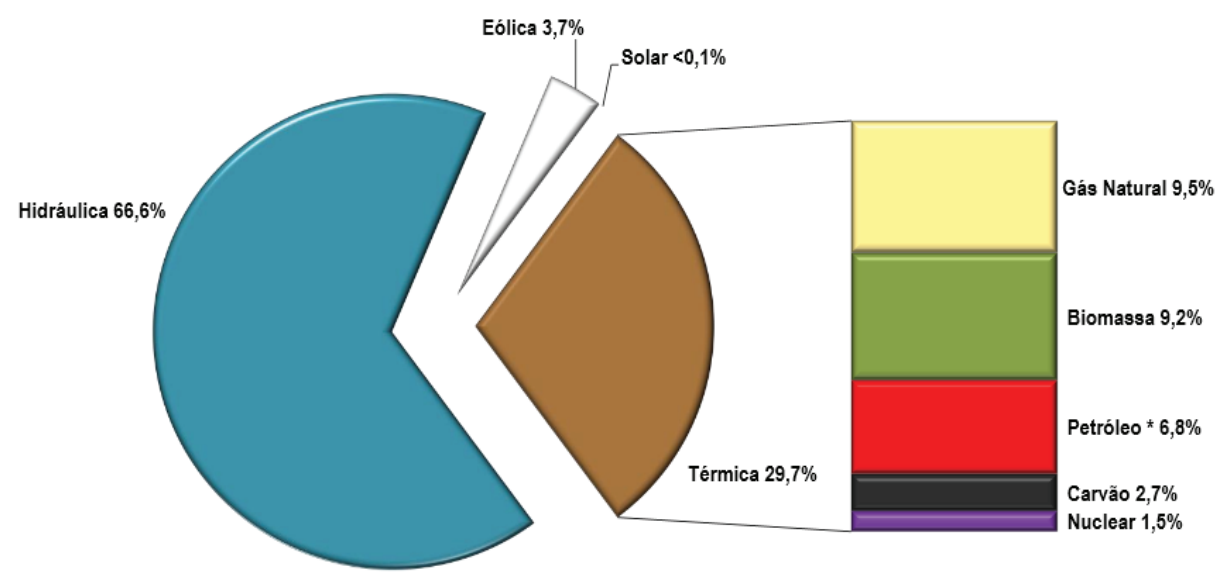

Fonte: Brasil, 2015. 
Segundo a Associação Brasileira de Energia Eólica (ABBEólica), os principais estados brasileiros produtores de energia eólica são os que estão listados na Tabela 1.

Tabela 1 - Descrição das unidades tomadoras de decisão (UTDs)

\begin{tabular}{lc}
\hline Estado & Potência instalada (MW) \\
\hline Rio Grande do Norte & $2.104,35$ \\
Ceará & $1.301,63$ \\
Rio Grande do Sul & $1.299,78$ \\
Bahia & 959,29 \\
Santa Catarina & 224,10 \\
Pernambuco & 101,15 \\
Piauí & 88,00 \\
Paraíba & 59,45 \\
Sergipe & 34,50 \\
Rio de Janeiro & 28,05 \\
Paraná & 11,50 \\
\hline Total & $6.211,80$ \\
\hline
\end{tabular}

Conforme a Tabela 1, o Estado do Rio Grande do Norte, com uma potência instalada de aproximadamente 2,1 GW, apresenta-se como o maior produtor dessa fonte renovável, sendo seguido por Ceará e o Rio Grande do Sul.

Logo, a pesquisa proposta tem a intenção de ordenar os estados produtores de energia eólica, de acordo com a medida de sua eficiência relativa, obtida por meio da aplicação de um modelo de análise por envoltória de dados. Dessa forma, as unidades a serem avaliadas, ou UTDs, estão descritas na Tabela 2.

Tabela 2 - Descrição das unidades tomadoras de decisão (UTDs).

\begin{tabular}{lcc}
\hline UTD & Estado & Região \\
\hline UDT1 & Bahia & Nordeste \\
UTD2 & Ceará & Nordeste \\
UTD3 & Paraíba & Nordeste \\
UTD4 & Pernambuco & Nordeste \\
UTD5 & Piaúi & Nordeste \\
UTD6 & Rio Grande do Norte & Nordeste \\
UTD7 & Sergipe & Nordeste \\
UTD8 & Rio de Janeiro & Sudeste \\
UTD9 & Paraná & Sul \\
UTD10 & Rio Grande do Sul & Sul \\
UTD11 & Santa Catarina & Sul \\
\hline
\end{tabular}

Em seguida, são descritos os fatores de avaliação, entre insumos e produtos, que serão utilizados para determinar a eficiência relativa das UTDs propostas nesta pesquisa.

\subsection{Fatores de avaliação}

Os dados obtidos para esta pesquisa são referentes à produção de energia elétrica, a partir dos parques eólicos instalados nos principais estados brasileiros, listados na Tabela 2, durante o ano de 2014. Esses dados foram usados para compor o conjunto de fatores que avaliam o desempenho de cada UTD proposta e são descritos no Quadro 1. 
Quadro 1 - Fatores usados para a avaliação da eficiência das UTDs.

\begin{tabular}{|l|l|}
\hline \multicolumn{1}{|c|}{ Insumos } & \multicolumn{1}{|c|}{ Produtos } \\
\hline $\begin{array}{l}\text { - Quantidade de turbinas eólicas (QTE) } \\
\text { • Potência eólica instalada em MW/mês (PEI) }\end{array}$ & • Potência elétrica gerada em MW/mês (PEG) \\
\hline
\end{tabular}

Dessa forma, são considerados como insumos a quantidade de turbinas eólicas, indicada por QTE, e a potência eólica instalada em MW/mês, indicada por PEI, enquanto o único produto será a potência elétrica gerada em MW/ mês, indicada por PEG. Será considerada nesta pesquisa a perspectiva de avaliação de eficiência das UTDs orientadas a produto, de modo que seja possível avaliar a capacidade de maximizar a produção de eletricidade com os insumos que representam o potencial eólico disponível.

A variável QTE é responsável pela conversão da energia cinética dos ventos em energia elétrica, enquanto a variável PEI evidencia a dimensão de cada estado e relaciona-se diretamente com a sua capacidade média de gerar eletricidade durante o ano de 2014. O único produto, a variável PEG, energia elétrica gerada, representa a quantidade de energia média produzida em todo o estado durante o ano de 2014.

É possível, então, afirmar que as UTDs apresentam a característica de possuírem tamanhos variados, implicando que elas tendem a ter rendimentos de escalas variáveis. Dessa forma, o método AED, selecionado para medir as eficiências, será o modelo BCC (retornos de escala variáveis), orientado a produto.

\subsection{Modelo AED e a fronteira invertida}

Assim, considerando $\mathrm{X}_{10}$ e $\mathrm{X}_{20}$ os valores dos insumos, e $\mathrm{Y}_{10}$ o valor do produto de cada $\mathrm{UTD}_{\mathrm{O}}$, o problema de programação linear a ser resolvido nesta pesquisa é dado da seguinte forma:

$$
\operatorname{minh} \mathrm{h}_{\mathrm{O}}=\mathrm{V}_{1} \mathrm{X}_{10}+\mathrm{V}_{2} \mathrm{X}_{20}+\mathrm{V}_{*}
$$

Sujeito a:

$$
\begin{aligned}
& \mathrm{U}_{1} \mathrm{Y}_{10}=1 \\
& \mathrm{U}_{1} \mathrm{Y}_{1 \mathrm{k}}-\mathrm{V}_{1} \mathrm{X}_{1 \mathrm{~K}}-\mathrm{V}_{2} \mathrm{X}_{2 \mathrm{~K}}+\mathrm{V}_{*} \leq 0, \quad(\mathrm{k}=1, \ldots, 11) \\
& \mathrm{U}_{1}, \mathrm{~V}_{1} \mathrm{eV} \mathrm{V}_{2} \geq 0 ; \mathrm{V}_{*} \in \mathbb{R}
\end{aligned}
$$

Em que $V_{1}$ e $V_{2}$ são os pesos dos insumos, e $U_{1}$ é o peso do único produto.

Para Ali (1993), os modelos AED geralmente resultam em um grande número de unidades eficientes. Além disso, devido às características matemáticas do modelo $\mathrm{BCC}$, as UTDs que apresentam o menor valor de um dos insumos ou o maior valor de um dos produtos são consideradas como eficientes, sendo chamadas de falsamente eficientes.

Um método que pode ser usado com a finalidade de eliminar as UTDs falsamente eficientes no modelo BCC é o da fronteira invertida, o qual consiste simplesmente em considerar os produtos como insumos e os insumos como produto para cada unidade; em seguida, resolve-se o modelo BCC do AED normalmente, com uma orientação a insumo.

Conforme Angulo Meza et al. (2007), a fronteira invertida tem o objetivo de construir um índice de eficiência composta, definido como sendo a média aritmética entre a eficiência em relação à fronteira AED convencional, $\mathrm{Ef}_{\text {pad }}$, e complemento da eficiência em relação à fronteira invertida, $\mathrm{Ef}_{\text {inv }}$, da seguinte forma:

$$
\mathrm{Ef}_{\text {comp }}=\frac{\mathrm{Ef}_{\text {conv }}+\left(1-\mathrm{Ef}_{\text {inv }}\right)}{2}
$$

"Para se obter um índice em que as unidades eficientes têm o valor de 1, é feita a normalização da eficiência composta, ao dividirem-se seus valores pela maior de todas as medidas de eficiência compostas." (Angulo Meza et al., 2007).

Para a perspectiva da fronteira invertida, a unidade mais eficiente será aquela que conseguir ter um desempenho mais equilibrado, isto é, que conseguir produzir muito de todos os seus produtos e gastar pouco de todos os seus insumos, sem se destacar em nenhum especificamente. Pode-se afirmar que a metodologia objetiva excluir dos grupos de UTDs eficientes as unidade falsamente eficientes (Almeida et al., 2007). 
É importante salientar que, com a aplicação da metodologia da fronteira invertida, também é possível ordenar as UTDs avaliadas de acordo com o valor da medida da eficiência composta normalizada, indicada por $\mathrm{Ef}_{\text {nor }}$.

\section{RESULTADOS E DISCUSSÃO}

\subsection{Análise da eficiência}

Todos os cálculos de eficiência para os parques eólicos foram realizados com auxílio da ferramenta computacional SIAD (Sistema Integrado de Apoio à Decisão), descrito em Angulo Meza et al. (2005). O SIAD deve ser utilizado em uma plataforma Windows e permite trabalhar com até 150 unidades e 20 variáveis (insumos + produtos), apresentando duas formas de entradas de dados: diretamente no programa, utilizando uma grade de entrada vazia, ou através de um arquivo txt.

Segundo Angulo Meza et al. (2005), uma vez que na literatura raramente se encontram aplicações como mais de 100 unidades a serem avaliadas e que a maioria das aplicações práticas trabalha com no máximo 10 variáveis, então, pode-se considerar suficiente esses números. Na Tabela 3, são apresentados os valores dos insumos QTE e PEI, e do produto PEG para cada um das UTDs avaliadas nesta pesquisa.

Tabela 3 - Dados de insumos e produtos para cada UTD da pesquisa.

\begin{tabular}{lccc}
\hline UTD & QTE & PEI (MW/mês) & PEG (MW/mês) \\
\hline UDT1 (Bahia) & 456 & 514,32 & 201,00 \\
UTD2 (Ceará) & 500 & $1.066,33$ & 405,04 \\
UTD3 (Paraíba) & 73 & 59,45 & 16,87 \\
UTD4 (Pernambuco) & 34 & 34,57 & 7,43 \\
UTD5 (Piauí) & 45 & 64,67 & 30,40 \\
UTD6 (Rio Grande do Norte) & 881 & $1.199,09$ & 396,13 \\
UTD7 (Sergipe) & 17 & 34,50 & 7,43 \\
UTD8 (Rio de Janeiro) & 14 & 28,05 & 8,96 \\
UTD9 (Paraná) & 5 & 11,50 & 2,73 \\
UTD10 (Rio Grande do Sul) & 580 & 720,80 & 183,85 \\
UTD11 (Santa Catarina) & 172 & 222,00 & 48,44 \\
\hline
\end{tabular}

Os dados que compõe a Tabela 3 se referem exclusivamente ao ano de 2014 (de janeiro à dezembro) e foram obtidos juntos à ABEEólica. Em seguida, aplicando então o modelo BCC com orientação a produto, determina-se a medida da eficiência relativa (eficiência convencional), para cada unidade avaliada. Em seguida, tem-se na Tabela 4 a medida da eficiência relativa, também chamada na pesquisa de eficiência convencional, para cada unidade avaliada.

Tabela 4 - Medidas das eficiências convencionais das unidades

\begin{tabular}{lc}
\hline UTD & Ef $_{\text {conv }}$ \\
\hline UDT1 (Bahia) & 1,0000 \\
UTD2 (Ceará) & 1,0000 \\
UTD3 (Paraíba) & 0,6094 \\
UTD4 (Pernambuco) & 0,5042 \\
UTD5 (Piauí) & 1,0000 \\
UTD6 (Rio Grande do Norte) & 0,9780 \\
UTD7 (Sergipe) & 0,6143 \\
UTD8 (Rio de Janeiro) & 0,9287 \\
UTD9 (Paraná) & 1,0000 \\
UTD10 (Rio Grande do Sul) & 0,6629 \\
UTD11 (Santa Catarina) & 0,5389 \\
\hline
\end{tabular}


Por meio da Tabela 4, é possível observar que, das 11 unidades avaliadas, quatro são consideradas eficientes e sete não eficientes. Dessa forma, pode-se afirmar que as unidades UTD1 (Bahia), UTD2 (Ceará), UTD5 (Piauí) e UTD9 (Paraná) são consideradas como os benchmarks do conjunto analisado. Ou seja, a produção de energia eólica para geração de eletricidade nos estados da Bahia, Ceará, Piauí e Paraná apresentaram medidas de eficiência igual a 100\%.

É possível determinar, através da Tabela 4, que a produção de energia eólica na geração de eletricidade dos 11 principais estados brasileiros se apresenta com uma eficiência relativa média de aproximadamente $80,33 \%$. Em seguida, na Figura 4, são apresentadas, de forma gráfica, as unidades avaliadas, em ordem decrescente, conforme a medida da sua eficiência convencional.

Figura 4 - Ordenação das UTDs avaliadas conforme a medida de sua eficiência convencional.

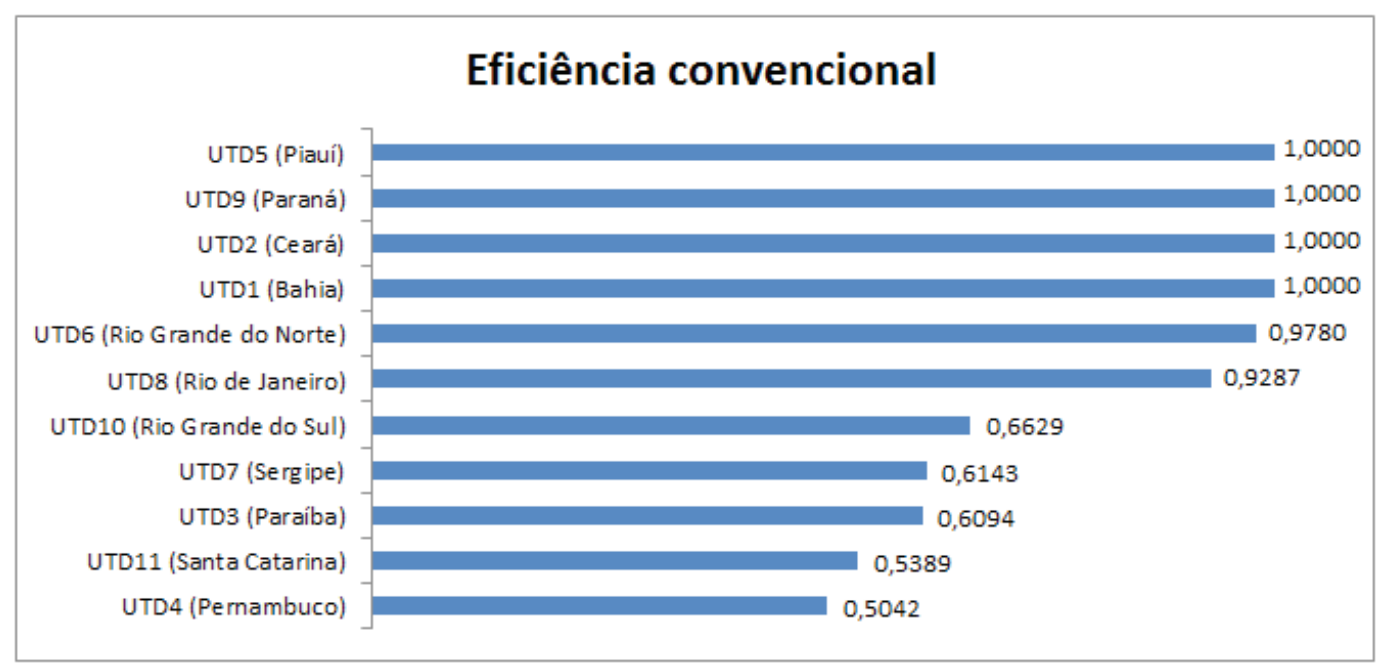

Das unidades não eficientes, destaca-se a produção eólica nos estados do Rio Grande do Norte, indicada por UTD6, e do Rio de Janeiro, indicada por UTD8, que apresentaram eficiências iguais a 97,80\% e 92,87\%, respectivamente, necessitando possivelmente de pequenos ajustes para atingirem a fronteira eficiente. Negativamente, destaca-se a produção de energia eólica em Pernambuco, indicada por UTD4, que apresentou a menor medida de eficiência de todo o conjunto, sendo igual a 50,42\%.

Analisando os dados das Tabelas 3 e 4, é possível ainda afirmar que a produção de energia eólica nos estados do Paraná, indicada por UTD9, e do Ceará, indicada por UTD2, apresentam uma falsa eficiência. Enquanto a UTD9 apresenta o insumo QTE igual a 5, que é o menor valor desse insumo dentre todas as unidades, a UTD2 apresenta o produto PEG igual a 405,04, que é o maior valor desse produto dentre todas as unidades.

\subsection{Fronteira invertida}

Aplicando, então, a metodologia da fronteira invertida, é possível montar a Tabela 5, que apresenta as eficiências convencional $\left(\mathrm{Ef}_{\text {conv }}\right)$, invertida $\left(\mathrm{Ef}_{\text {inv }}\right)$, composta $\left(\mathrm{Ef}_{\text {comp }}\right)$ e normalizada $\left(\mathrm{Ef}_{\text {nor }}\right)$ para cada unidade avaliada, de modo que seja possível ordená-las e eliminar as falsamente eficientes. É importante salientar que a eficiência composta, Ef comp, foi obtida por meio da equação 15 , enquanto a eficiência normalizada, $\mathrm{Ef}_{\text {nor }}$, foi obtida pela divisão de $\mathrm{Ef}_{\text {comp }}$ pela máxima medida da eficiência composta, que para o conjunto analisado foi dada por 0,7695 , referente à UTD5 (Piauí). 
Tabela 5 - Medidas das eficiências das unidades avaliadas

\begin{tabular}{lcccc}
\hline UTD & $\mathrm{Ef}_{\text {conv }}$ & $\mathrm{Ef}_{\text {inv }}$ & $\mathrm{Ef}_{\text {comp }}$ & Ef $_{\text {nor }}$ \\
\hline UDT1 (Bahia) & 1,0000 & 0,7099 & 0,6451 & 0,8383 \\
UTD2 (Ceará) & 1,0000 & 0,8325 & 0,5838 & 0,7587 \\
UTD3 (Paraíba) & 0,6094 & 1,0000 & 0,3047 & 0,3960 \\
UTD4 (Pernambuco) & 0,5042 & 1,0000 & 0,2521 & 0,3276 \\
UTD5 (Piaú́) & 1,0000 & 0,4611 & 0,7695 & 1,0000 \\
UTD6 (Rio Grande do Norte) & 0,9780 & 1,0000 & 0,4890 & 0,6355 \\
UTD7 (Sergipe) & 0,6143 & 0,9981 & 0,3081 & 0,4004 \\
UTD8 (Rio de Janeiro) & 0,9287 & 0,6810 & 0,6238 & 0,8108 \\
UTD9 (Paraná) & 1,0000 & 1,0000 & 0,5000 & 0,6498 \\
UTD10 (Rio Grande do Sul) & 0,6629 & 1,0000 & 0,3315 & 0,4308 \\
UTD11 (Santa Catarina) & 0,5389 & 1,0000 & 0,2694 & 0,3502 \\
\hline
\end{tabular}

Em seguida, na Figura 5, são apresentadas, de forma gráfica, as unidades avaliadas, em ordem decrescente, conforme a medida da sua eficiência normalizada, apresentando o índice de eficiência.

Figura 5 - Ordenação das UTDs avaliadas conforme a medida de sua eficiência normalizada.

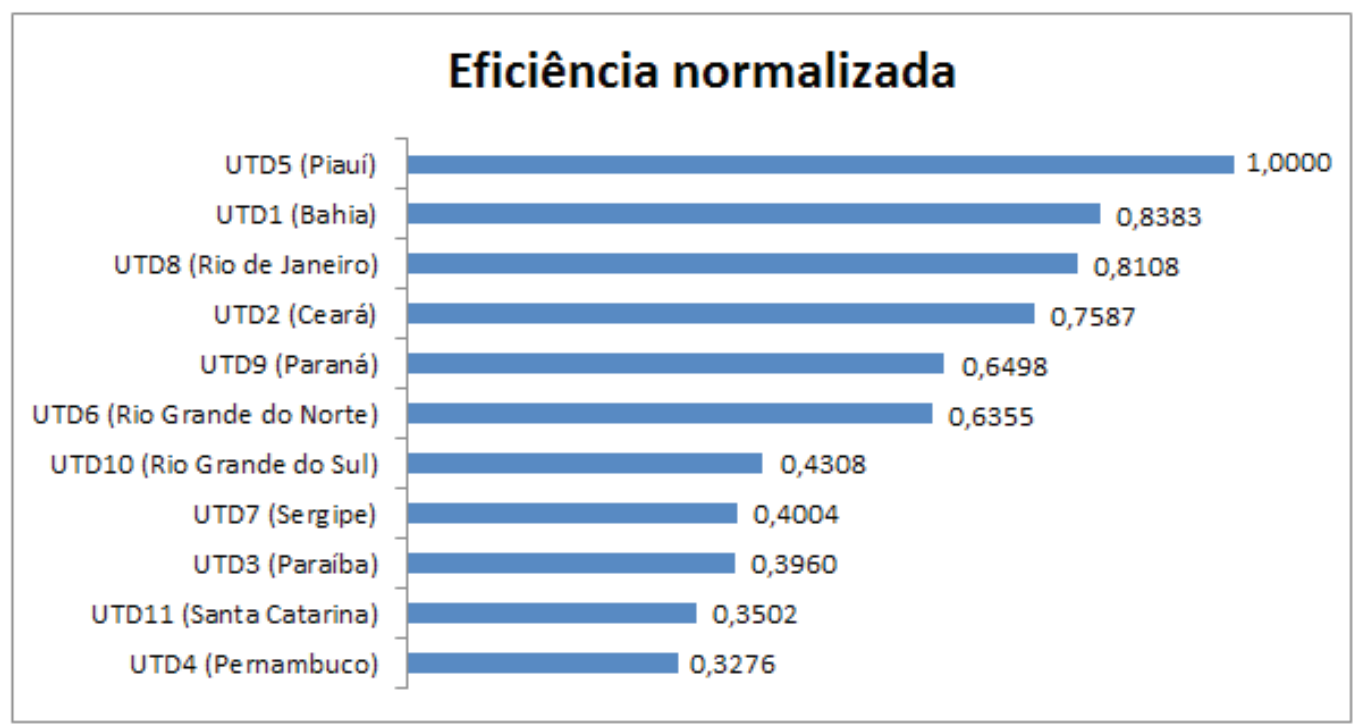

Conforme os dados apresentados, pode-se concluir que a conversão de energia eólica em eletricidade, no ano de 2014, no Piauí, representou a unidade mais eficiente dentre todo o conjunto analisado na pesquisa.

Também foi possível concluir que as unidades UTD2 (Ceará) e UTD9 (Paraná) são realmente unidades falsamente eficientes. Isto é, as produções de energia eólica do Ceará e do Paraná, consideradas eficientes, passam a ocupar a quarta e a quinta posição, respectivamente, de acordo com o índice de eficiência, definido pela eficiência normalizada. Vale salientar que a produção de energia eólica do Estado do Rio de Janeiro, com esse índice, passa a ocupar a terceira posição, ultrapassando o Ceará e o Paraná.

Por fim, a UTD4, referente à produção de energia eólica no Estado de Pernambuco, destacou-se negativamente, com um índice de eficiência igual a 32,76\%, sendo a unidade com a menor eficiência do conjunto analisado, confirmando o resultado obtido anteriormente. Além de Pernambuco, que ocupa a última posição, os estados do Rio Grande do Sul, UTD10, de Sergipe, UTD7, da Paraíba, UTD3, e de Santa Catarina, UTD11, também se mantiveram na mesma posição. 


\subsection{Conjuntos de referências e projeções a serem alcançadas}

Cada uma das benchmarks do conjunto, listadas na seção anterior, pode fazer parte do grupo de referência das unidades não eficientes. Na Tabela 6, são apresentados os conjuntos de referência para cada UTD ineficiente da pesquisa.

Tabela 6 - Conjunto de referência das UTDs não eficientes.

\begin{tabular}{l|l|c|c}
\hline \multicolumn{2}{|c|}{ UTDs não eficientes } & UTDs de referência & Peso \\
\hline $5^{\text {a }}$ & UTD6 (Rio Grande do Norte) & UTD2 (Ceará) & 1,0000 \\
\hline $6^{\text {a }}$ & UTD8 (Rio de Janeiro) & UTD9 (Paraná) & 0,9065 \\
\hline & & UTD5 (Piauí) & 0,0820 \\
\hline & & UTD2 (Ceará) & 0,0115 \\
\hline $7^{\text {a }}$ & UTD10 (Rio Grande do Sul) & UTD1 (Bahia) & 0,6259 \\
\hline & & UTD2 (Ceará) & 0,3741 \\
\hline $8^{\text {a }}$ & UTD7 (Sergipe) & UTD9 (Paraná) & 0,9020 \\
\hline & & UTD5 (Piauí) & 0,0802 \\
\hline & & UTD2 (Ceará) & 0,0178 \\
\hline $9^{\text {a }}$ & UTD3 (Paraíba) & UTD5 (Piauí) & 0,9018 \\
\hline & & UTD9 (Paraná) & 0,0982 \\
\hline $10^{\text {a }}$ & UTD11 (Santa Catarina) & UTD5 (Piauí) & 0,6949 \\
\hline & & UTD1 (Bahia) & 0,2686 \\
\hline & & UTD2 (Ceará) & 0,0365 \\
\hline $11^{\text {a }}$ & UTD4 (Pernambuco) & UTD9 (Paraná) & 0,5661 \\
\hline & & UTD5 (Piauí) & 0,4339 \\
\hline
\end{tabular}

É importante salientar que quanto maior for a frequência que uma unidade eficiente aparece no grupo de referência, mais alta é a possibilidade do seu desempenho ser de excelência.

No Quadro 2, são apresentadas as projeções (alvos) a serem alcançadas por cada fator de insumo e de produto de forma que uma UTD considerada ineficiente atinja a eficiência máxima (igual à unidade). Essas melhorias sugerida nos fatores de uma determinada UTD, objetivam que a mesma otimize a sua relação insumo/produto. É importante ressaltar que as projeções definidas nos insumos e produtos de uma unidade ineficiente são apenas sugestões e, muitas vezes, na prática, a aplicação dessas sugestões não é viável.

A produção de energia eólica no Estado de Pernambuco, que apresenta a menor medida de eficiência, para se tornar eficiente, deve reduzir o número de turbinas eólicas em operação de 34 para 22, aumentando a potência gerada ao mês de 7,43 MW para 14,74 MW, mantendo a mesma potência instalada em todo o estado.

Para isso ser possível, é necessário trocar as turbinas atuais por 22 turbinas eólicas mais potentes que em conjunto apresentem uma potência instalada de 34,57 MW. Assim, devido aos custos do equipamento e de sua montagem em parques eólicos, essa troca é economicamente inviável para o estado. Porém, como a vida útil de um aerogerador é em torno de 20, essa informação poderia ser útil nas substituições dos equipamentos.

Semelhantemente, os estados do Rio Grande do Sul e da Paraíba devem substituir os aerogeradores atuais por uma quantidade menor de máquinas, de maior potência, mantendo a mesma potência instalada em todo o estado, e aumentar a produção de potência elétrica. Da mesma forma que em Pernambuco essa sugestão é economicamente inviável. 
Quadro 2 - Projeção a ser alcançada pelos fatores de cada UTD não eficiente.

\begin{tabular}{|c|c|c|c|}
\hline \multicolumn{4}{|c|}{$5^{\text {a) }}$ UTD6 (Rio Grande do Norte) - 0,9780} \\
\hline \multirow[b]{2}{*}{ Atual } & QTE & PEI & PEG \\
\hline & 881 & $1.199,09$ & 396,13 \\
\hline Projeção & 500 & $1.066,33$ & 405,04 \\
\hline \multicolumn{4}{|c|}{ 6) UTD8 (Rio de Janeiro) - 0,9287 } \\
\hline & QTE & PEI & PEG \\
\hline Atual & 14 & 28,05 & 8,96 \\
\hline Projeção & 14 & 28,05 & 9,65 \\
\hline \multicolumn{4}{|c|}{$7^{\text {a })}$ UTD10 (Rio Grande do Sul) - 0,6629 } \\
\hline & QTE & PEI & PEG \\
\hline Atual & 580 & 720,80 & 183,85 \\
\hline Projeção & 472 & 720,80 & 277,32 \\
\hline \multicolumn{4}{|c|}{ 8a) UTD7 (Sergipe) - 0,6143 } \\
\hline & QTE & PEI & PEG \\
\hline Atual & 17 & 34,50 & 7,43 \\
\hline Projeção & 17 & 34,50 & 12,09 \\
\hline \multicolumn{4}{|c|}{ 9a) UTD3 (Paraíba) - 0,6094 } \\
\hline & QTE & PEI & PEG \\
\hline Atual & 73 & 59,45 & 16,87 \\
\hline Projeção & 41 & 59,45 & 27,68 \\
\hline \multicolumn{4}{|c|}{ 10'a UTD11 (Santa Catarina) - 0,5389 } \\
\hline & QTE & PEI & PEG \\
\hline Atual & 172 & 222,00 & 48,44 \\
\hline Projeção & 172 & 222,00 & 89,90 \\
\hline \multicolumn{4}{|c|}{ 11 ${ }^{\text {a })}$ UTD4 (Pernambuco) - 0,5042 } \\
\hline & QTE & PEI & PEG \\
\hline Atual & 34 & 34,57 & 7,43 \\
\hline Projeção & 22 & 34,57 & 14,74 \\
\hline
\end{tabular}

Em resumo, conforme os dados apresentados no Quadro 2, as unidades não eficientes, em geral, atingem a fronteira invertida com a redução do insumo QTE (quantidade de turbinas eólicas em operação no estado) e um aumento do produto PEG (potência elétrica gerada ao mês em todo o estado).

\section{Conclusões}

Este trabalho analisou a eficiência dos principais estados produtores de energia eólica no que diz respeito à conversão de energia dos ventos em energia elétrica, levando em consideração o processo de produção. Para isso, foi usado o modelo BCC, com uma orientação a produto. 
A análise inicial indicou que, das onze unidades avaliadas, quatro são classificadas como eficientes; a saber: UTD1 (Bahia), UTD2 (Ceará), UTD5 (Piauí) e UTD9 (Paraná). Em seguida, a metodologia da fronteira invertida indicou que as produções de energia eólica dos estados do Ceará e Paraná são classificadas como falsamente eficientes.

A produção de energia eólica no Estado do Piauí, no período considerado, apresentou-se com um desempenho de excelência, sendo a unidade mais eficiente do conjunto analisado. Além disso, sua frequência em conjunto de referência nas unidades não eficientes foi igual a cinco. Por outro lado, a produção de energia eólica no Estado de Pernambuco em 2014 se apresentou com o maior índice de referência em relação aos onze estados analisado.

Em regra, as alterações propostas para as unidades ineficientes do conjunto analisado consistem em reduzir os valores dos insumos representativos e elevar o valor do único produto.

Finaliza-se este trabalho de pesquisa com a afirmação de que o uso da análise por envoltória de dados pode auxiliar os gestores na avaliação da produção de energia eólica, permitindo assim uma melhor gestão do recurso eólico para obter energia elétrica.

\section{Referências}

ADLER, N.; YAZHEMSKY, E. Improving discrimination in data envelopment analysis: PCA-DEA or variable reduction. European Journal of Operational Research, n.202, pp. 273-284, 2010.

ALI, A. I. Streamlined computation for data envelopment analysis. European Journal of Operational Research, $n$. 64, pp. 61-67, 1993.

ALMEIDA, M. R. de; MARIANO, E. B.; REBELATTO, D. A. do N. Análise de eficiência dos aeroportos internacionais. Revista Produção, v. 7, n. 4, pp. 9-26, 2007.

ANGULO MEZA, L. et al. ISYDS: Integrated System for Decision Support (SIAD - Sistema Integrado de Apoio à Decisão): A software package for data envelopment analysis model. Pesquisa Operacional, v. 25, n. 3, pp. 493-503, 2005.

ATHANASSOPOULOS, A. D. Discriminating among relatively efficient units in data envelopment analysis: a comparison of alternative methods and some extensions. American Journal of Operations Research, n. 2, pp. 1-9, 2012 .

BANKER, R. D. Maximum likelihood, consistency and data envelopment analysis: a statistical foundation. Management Science, v. 39, n. 10, p. 1265-1273, 1993.

BANKER, R. D.; CHARNES, A.; COOPER, W. W. Some models for estimating technical and scale inefficiencies in data envelopment analysis. Management Science, v. 30, n. 9, pp. 1078-1092, 1984.

BARBOSA, R. de P.; BASTOS, A. P. V. Utilização da análise por envoltória de dados (DEA) na mensuração da eficiência das prestadoras de serviços de água e esgotamento sanitário: um enfoque no desempenho da companhia de saneamento do estado do Pará. Revista Economia e Gestão, v. 14, n. 35, p. 151-181, 2014.

BRASIL. Ministério de Minas e Energia. Secretaria de Energia Elétrica. Departamento de Monitoramento do Sistema Elétrico. Boletim mensal de monitoramento do sistema elétrico brasileiro - Janeiro 2015. Esplanada dos Ministérios - Bloco U $-6^{\circ}$ andar, Brasília - DF, 2015. Disponível em: $<$ http://www.mme.gov.br/mme/menu/todas publicacoes.html>. Acesso em: 16 de set., 2015.

CARNEIRO, F. O. M.; ROCHA, H. H. B.; ROCHA, P. A. C. Investigation of possible societal risk associated with wind power generation systems. Renewable and Sustainable Energy Reviews, n. 19, pp. 30-36, 2013.

CHARNES, A.; COOPER, W. W.; RHODES, E. Measuring the efficiency of decision making units. European Journal of Operational Research, v. 2, n. 6, pp. 429-444, 1978.

FADIGAS, E. A. F. A. Energia eólica. Barueri: Manole, 2011.

GOMES JÚNIOR, J. F.; SOARES DE MELLO, J. C. C. B.; ANGULO MEZA, L. DEA nonradial efficiency based on vector properties. International Transactions Inoperational Research, n. 20, pp. 341-364, 2013.

GOMES, E. G.; MELLO, J. C. C. B. S. de; FREITAS, A. C. R. de. Efficiency measures for a non-homogeneous group of family farmers. Revista Pesquisa Operacional, n.32, pp.561-574, 2012. 
HAMDAN, A. et al. A review on the micro energy harvester in structural health monitoring (SHM) of biocomposite material for vertical axis wind turbine (VAWT) system: a Malaysia perspective. Renewable and Sustainable Energy Reviews, n. 35, pp.23-30, 2014.

HANSEN, M. O. L. Aerodynamics of wind turbines. 2.ed.. USA: Earthscan, 2008.

KNIESS, C. T. et al. O uso da energia eólica no Brasil: aspectos econômico, social, ambiental e legal. Organizações e Sustentabilidade, v. 1, n. 1, p. 2-18, 2013.

MEZA, L. A. et al. Seleção de variáveis em DEA aplicada a uma análise do mercado de energia eléctrica. Investigação Operacional, n. 27, pp. 21-36, 2007.

NEUMEISTER, R. F.; SILVA, C. V. da. Análise do escoamento sobre uma turbina eólica vertical usando CFD. Revista Perspectiva, v. 38, n. 142, pp. 127-138, 2014.

PARK, J. L. et al. Comparing the efficiency and productivity of construction firms in China, Japan and Korea using DEA and DEA based malmquist. Journal of Asian Architectures and Building Engineering, n.14, v.1, pp.57-64, 2015.

PÉRICO, A. E.; REBALATTO, D. A. do N.; SANTANA, N. B. Eficiência bancária: os maiores bancos são os mais eficientes? Uma análise por envoltória de dados. Revista Gestão e Produção, v. 15, n. 2, p. 412-431, 2008.

PINTO, M. de O. Fundamentos de energia eólica. Rio de Janeiro : LTC, 2014.

PIRES, J. C.; OLIVEIRA, B. F. Modelagem e simulação virtual de pá para aerogerador de pequeno porte. Revista Design \& Tecnologia, v.1, n. 2., pp. 69-76, 2010.

RODRIGUES, M. V. S. Avaliação do desempenho da cobrança da água bruta por categoria de uso nas bacias do Estado do Ceará utilizando a análise por envoltória de dados. 2014. 174 f. Tese (Doutorado em Engenharia Civil) Universidade Federal do Ceará, Fortaleza - CE, 2014.

RODRIGUES, M. V. S.; AQUINO, M. D.; THOMAZ, A. C. F. Análise por envoltória de dados utilizada para medir o desempenho relativo da cobrança pelo uso da água nas bacias hidrográficas do estado do Ceará. Revista de Gestão de Águas da América Latina (REGA), v. 12, n. 1, p. 15-29, 2015.

SHAMSHIRBAND, S. et al. Wind turbine power coefficient estimation by soft computing methodologies: Comparative study. Energy Conversion and Management, n. 81, pp.520-526. 2014.

SHARMA, R.; PATEL, B. Design and simulation of Darrieus (Eggbeater) type vertical axis wind turbine using open source software Q blade. International Journal for Innovative Research in Science \& Technology, v.1, n.12, 2015.

Sobre os autores

\section{Marcus Vinícius Sousa Rodrigues}

Engenheiro Mecânico, Universidade Federal do Ceará - UFC. Mestre e Doutor em Engenharia Civil, ambos na área de concentração em Recursos Hídricos, pela Universidade Federal do Ceará - UFC. Professor Adjunto do Departamento de Ciências Exatas, Tecnológicas e Humanas - DCETH, campus de Angicos (Angicos, RN), da Universidade Federal Rural do Semi-Árido - UFERSA.

\section{Marisete Dantas de Aquino}

Engenheira de Pesca, Universidade Federal do Ceará - UFC. Mestre em Engenharia Civil, área de concentração em Recursos Hídricos, pela Universidade Federal do Ceará - UFC. Doutora em Meio Ambiente e Recursos Hídricos, pela École des Hautes Études en Sciences Sociales - EHESS, França. Professora Titular do Departamento de Engenharia Hidráulica e Ambiental - DEHA, campus do Pici (Fortaleza, CE), da Universidade Federal do Ceará - UFC.

\section{Antônio Clécio Fontelles Thomaz}

Matemático, Universidade Federal do Ceará - UFC. Mestre em Informática, pela Pontifícia Universidade Católica do Rio de Janeiro - PUC, RJ. Doutor em Engenharia de Sistema e Computação, pela Universidade Federal do Rio de Janeiro - UFRJ. Professor Adjunto do Centro de Ciências e Tecnologia, campus do Itaperi (Fortaleza, CE), da Universidade Estadual do Ceará - UECE. 